\title{
Transient inhomogeneous flow patterns in supercooled liquids under shear
}

Article

Accepted Version

Fuereder, I. and Ilg, P. (2017) Transient inhomogeneous flow patterns in supercooled liquids under shear. Soft Matter, 13 (11). pp. 2192-2200. ISSN 1744-683X doi: https://doi.org/10.1039/C7SM00178A Available at https://centaur.reading.ac.uk/69267/

It is advisable to refer to the publisher's version if you intend to cite from the work. See Guidance on citing.

Published version at: http://pubs.rsc.org/en/content/articlelanding/2017/sm/c7sm00178aflldivAbstract To link to this article DOI: http://dx.doi.org/10.1039/C7SM00178A

Publisher: Royal Society of Chemistry

All outputs in CentAUR are protected by Intellectual Property Rights law, including copyright law. Copyright and IPR is retained by the creators or other copyright holders. Terms and conditions for use of this material are defined in the End User Agreement.

\section{www.reading.ac.uk/centaur}

\section{CentAUR}

Central Archive at the University of Reading

Reading's research outputs online 


\section{Transient inhomogeneous flow patterns in supercooled liquids under shear}

Ingo Fuereder*1 and Patrick $\mathrm{Ig}^{2}$

1) ETH Zurich, Department of Materials, Vladimir-Prelog-Weg 1-5/10, CH-8093 Zurich.

${ }^{2)}$ School of Mathematical, Physical and Computational Sciences, University of Reading, Reading RG6 6AX.

(Dated: February 23, 2017)

Supercooled liquids and other soft glassy systems show characteristic spatial inhomogeneities in their local dynamical properties. Using detailed molecular simulations, we find that for sufficiently low temperatures and sufficiently high shear rates supercooled liquids also show transient, inhomogeneous flow patterns (shear banding) in start-up of steady shear flow, similar to what has already been observed for many other soft glassy systems. We verify that the onset of transient shear banding coincides quite well with the appearance of a stress overshoot for temperatures in the supercooled regime. We find that the slower bands adapt less well to the imposed deformation and therefore accumulate higher shear stresses compared to the fast bands at comparable local shear rates. Our results also indicate that the shear rates of the fast and slow bands are adjusted such that the local dissipation rate is approximately the same in both bands. 


\section{INTRODUCTION}

A huge class of various different materials such as microgels, pastes, slurries, foams, dense suspensions, supercooled liquids etc. are usually subsumed as soft glassy materials sharing the hallmarks of glass physics, i.e. structural disorder and metastability. Recent progress in the field has shown that not only slow relaxation processes are characteristic of these systems but also strong spatial variations in local dynamical properties, so-called dynamical heterogeneities ${ }^{1-4}$. There is evidence from extensive experimental and theoretical investigations that glassy systems also show a common phenomenology when subjected to external flow $^{5-7}$. However, the existence and role of spatial inhomogeneities in driven glassy systems is currently a matter of debate. Extended mode-coupling theory, e.g., successfully predicts many rheological properties including stress overshoots of glassy systems assuming spatially homogeneous systems ${ }^{8-12}$.

A range of glassy materials including amorphous solids show steady-state shear banding ${ }^{13,14}$, i.e. the occurrence of a high and low shear rate band in the stationary velocity profile that develops in response to an externally applied steady shear deformation with rate $\dot{\gamma}^{15}$. Stationary shear banding is usually associated with a mechanical instability of the underlying constitutive relation, but is not observed in other soft glassy materials that do not show aging or thixotropy. Instead, experiments on so-called simple yield stress fluids including foams, emulsions and gels observed transient shear bands that form during start-up of steady shear but do not persist to steady state ${ }^{16-18}$. These findings are in agreement with the observation that these soft glassy materials have a monotonic constitutive curve.

Supercooled liquids as an important model system for glassy physics show neither aging nor a yield stress above a critical temperature. Notwithstanding these differences, do supercooled liquids show transient shear banding similar to simple yield stress fluids? Presently, most theories on transient shear banding offer one of the following three explanations of the phenomenon: an instability of the dynamical equations, a time dependent flow curve being non-monotonic on an intermediate time-scale and an mechanical instability associated with a stress overshoot frequently occurring in the stress-strain curve. ${ }^{19,20}$

Extensive research has been carried out both numerically and theoretically on the timedependent formation of shear bands in the transient regime and their possible extension to steady state within the framework of the so-called shear transformation zone model 
employing effective-temperature thermodynamics ${ }^{21-24}$, within the fluidity model $^{25}$, in an alternative mesoscopic approach by Jagla ${ }^{26,27}$ and within the phenomenological soft-glassy rheology model ${ }^{28-30}$. We note that a general criterion for the occurrence of transient shear banding has been proposed by Moorcroft and Fielding ${ }^{31}$, where the authors predict that the onset of shear banding occurs shortly before the stress overshoot, with a correction term depending on the form of the stress-strain curve.

In previous studies molecular dynamics (MD) simulations have been proven to be useful to investigate shear-band formation in polymer melts ${ }^{32}$ and solid glasses ${ }^{13,33,34}$ where simulation conditions have been set up such that no bias on the velocity profile was imposed. In this letter we present results from extensive MD simulations of a prototypical model of a supercooled liquid in start-up of steady shear, clarifying the presumed connection between the occurrence of transient shear banding in supercooled liquids with the presence of a stress overshoot. We also show that the slow band is structurally different from the fast band, carrying higher shear stresses at comparable local shear rates as it has less well adapted to shear deformation.

\section{MODEL AND METHODS}

\section{A. Model}

As a model glass former, we choose the well-studied three-dimensional binary LennardJones fluid of Kob and Andersen ${ }^{35}$ with the interaction potential

$$
\phi_{i j}(r)=4 \epsilon_{i j}\left(\left(\frac{\sigma_{i j}}{r}\right)^{12}-\left(\frac{\sigma_{i j}}{r}\right)^{6}\right)
$$

where $\epsilon_{11}=1, \epsilon_{12}=1.5, \epsilon_{22}=0.5$ and $\sigma_{11}=1, \sigma_{12}=0.88, \sigma_{22}=0.8$. All particles have the same mass $m=1$. Lennard-Jones units are used throughout the article such that length is reported in units of $\sigma_{11}$, energy in units of $\epsilon_{11}$ and time in units of $\left(\sigma_{11}^{2} m / \epsilon_{11}\right)^{1 / 2}$. The ratio of large to small particles is 80:20, a cutoff radius $r_{c}=2.5$ was used and the potential was shifted such that it vanishes at $r_{c}$. Simulations were carried out with $N=8000$ particles, which were confined to a cubic box with length $L=18.8$. This corresponds to a density of $\rho=1.204$. Periodic boundary conditions were used in $x$ - and in $z$-direction. In $y$-direction the system was confined by a frozen-in layer of particles of type 1 which formed a fcc lattice with lattice constant $a=0.85$, which is small enough such that particles cannot penetrate 
wall. Molecular dynamics simulations are performed in the NVT-ensemble and we tested different methods of thermostatting by either employing a profile unbiased thermostat ${ }^{36}$ with velocity rescaling every 10 time steps or by using a DPD thermostat ${ }^{37,38}$ where the cut-off for the DPD interaction was chosen to be $r_{c}=2.5$. As a time step, we used $\Delta t=0.001$ for temperatures $T>0.48$ and $\Delta t=0.005$ for $T \leq 0.48$. All simulations were performed using the Large-scale Atomic/Molecular Massively Parallel Simulator ${ }^{39}$ package. For the ease of presentation we here show data for the DPD results only. Statistically independent samples are prepared at a high temperature then slowly cooled down into the supercooled regime and carefully equilibrated by waiting several relaxation times of the $\alpha$-relaxation process of the self-intermediate scattering function (see subsequent section). After equilibration, a constant shear rate $\dot{\gamma}=2 v_{0} / L$ is externally applied by moving the upper and lower layer with a constant velocity $+v_{0}$ and $-v_{0}$, respectively. This procedure is suitable to study shear banding since no velocity profile is imposed on the system as has been shown e.g. in the case of entangled polymer melts ${ }^{32}$. A snapshot of the simulation box is depicted in figure 1.

\section{B. Equilibration}

Independent starting configurations were created by randomly distributing the particles in the simulation box at density $\rho$. The system was equilibrated at a high temperature $(T=2.0)$ and slowly cooled down in the supercooled regime but above the mode coupling critical temperature $T_{c}=0.435 .{ }^{40}$ The systems were equilibrated by MD simulations run for $5-10 \alpha$-relaxation times of the self-intermediate scattering function

$$
F(\mathbf{q}, t)=\frac{1}{N}\left\langle\sum_{i=1}^{N} \exp \left(i \mathbf{q} \cdot\left(\mathbf{r}_{i}(t)-\mathbf{r}_{i}(0)\right)\right)\right\rangle
$$

evaluated at the $q=|\mathbf{q}|$ value which corresponds to the maximum of the static structure factor. We verify that the results do not depend on the waiting time $t_{w}$ by comparing the self-intermediate scattering function and the mean-square displacement of the particles $\left(\frac{1}{N} \sum_{i=1}^{N}\left(\mathbf{r}_{i}(t)-\mathbf{r}_{i}(0)\right)^{2}\right)$, for different waiting times $t_{w}=25000, t_{w}=100000, t_{w}=250000$. Here, $t_{w}$ is the time period during which the system has been simulated after equilibration but before data were collected. The appearance of a plateau in the mean-square displacement and $F(\mathbf{q}, t)$ followed by normal diffusion and decay of correlations is a typical behaviour found in supercooled liquids (see e.g. Ref. ${ }^{35}$ ). Moreover, contrary to the case of glasses and 


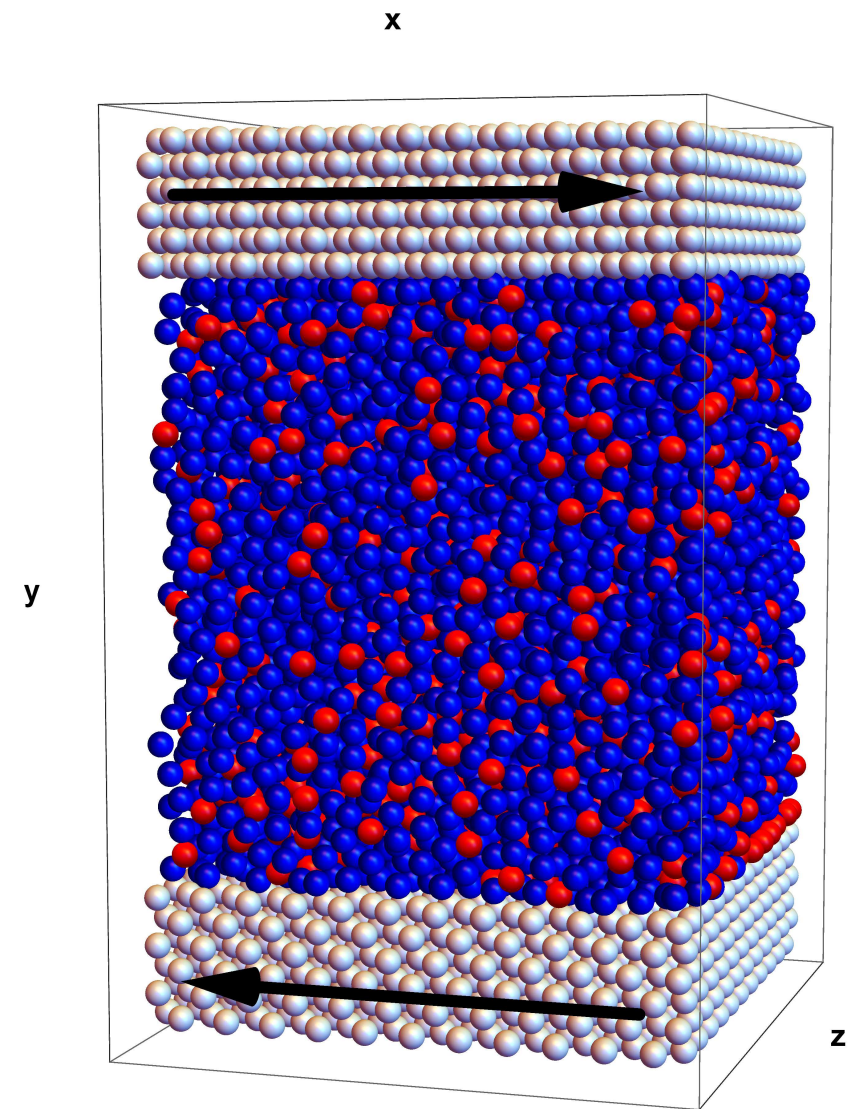

Figure 1. Snapshot of the simulation box. Particles of type one and two are shown in blue and red, respectively. Frozen-in particles forming the boundary in $y$-direction are colored light blue. Shearing is achieved by moving the frozen-in layers parallel to the black arrows with constant velocity $v_{0}$.

amorphous solids, the waiting time does not have a significant influence, neither on these quantities (see figure 2) nor on the results presented below.

\section{Start-up of steady shear}

After careful equilibration of numerous independent sample configurations as described in Sect. II B, steady shear is applied to the system at time $t=0$ by moving the upper and lower frozen-in layers of particles in $x$-direction with constant velocity $\pm v_{0}$, respectively. By extrapolating the resulting bulk velocity profile to the wall, we computed the slip length of the simulation to make sure that the results are not biased by wall-slip. No relevant slip was found for the temperature/shear-rate regime used in this work. We use a profile-unbiased 


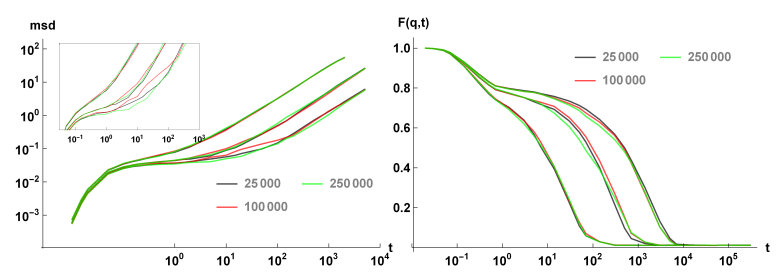

Figure 2. Left: Mean squared displacement of dominant particle type as a function of time for different waiting times. Temperatures are $T=0.60,0.50,0.46$ from top to bottom. Inset shows a zoom into intermediate time scales. Right: The self-intermediate scattering function $F(q, t)$, Eq. (2), as a function of time for the same conditions and waiting times as in the left panel.

thermostat or a dissipative particle thermostat to ensure constant temperature simulations. The instantaneous shear stress we evaluate during time evolution from the Irving-Kirkwood formula ${ }^{41}$

$$
\sigma_{x y}=\frac{1}{V} \sum_{i=1}^{N} m_{i} v_{i, x} v_{i, y}-\frac{1}{2 V} \sum_{i=1}^{N} \sum_{j=1}^{N} r_{i j, x} F_{i j, y},
$$

where $m_{i}$ is the particle mass, $v_{i, x}$ the peculiar velocity in $x$-direction, $V$ the system volume, $r_{i j, x}$ the $x$-component of the connector and $F_{i j, y}$ the $y$-component of the force vector between particles $i$ and $j$.

Typical stress-strain curves, i.e. shear stress $\sigma_{x y}$ versus strain $\gamma=t \dot{\gamma}$, obtained in a startup shear simulation are depicted in figure 3. We observe that the shear stress increases with decreasing temperature, developing a stress overshoot at sufficiently low temperatures. Similar stress-strain curves have been reported earlier ${ }^{12,40,42}$. As it is generic for viscoelastic fluids, the shear stress $\sigma_{x y}$ initially increases linearly with the strain but this rapid elastic stress increase competes with viscous relaxation processes. As these stress relaxation events become more frequent, they balance the elastic response accompanied by a decrease of the accumulated stress to level off to a stationary value. Similar stress strain curves are also observed for polymer gels and hairy nanoparticles ${ }^{43,44}$ where the drop in the stress-strain curve is associated with the breaking of bonds in the microstructure as a response to the applied force. Likewise, many complex fluids exhibit a well-defined microstructure (e.g. hydrogels, block copolymers) and structural changes can be accounted for their macroscopic deformation behaviour. Despite many efforts, the analogue of effective bonds and the relevant structural features in supercooled liquids are still unclear. Therefore, we note that it is possible that the microscopic mechanisms underlying stress-relaxation in supercooled 
liquids differ in their nature from those of other soft materials and that similar rheological properties may nevertheless appear on a macroscopic level, where details of the microscopic mechanisms are less relevant compared to the common features of structural disorder and metastability.

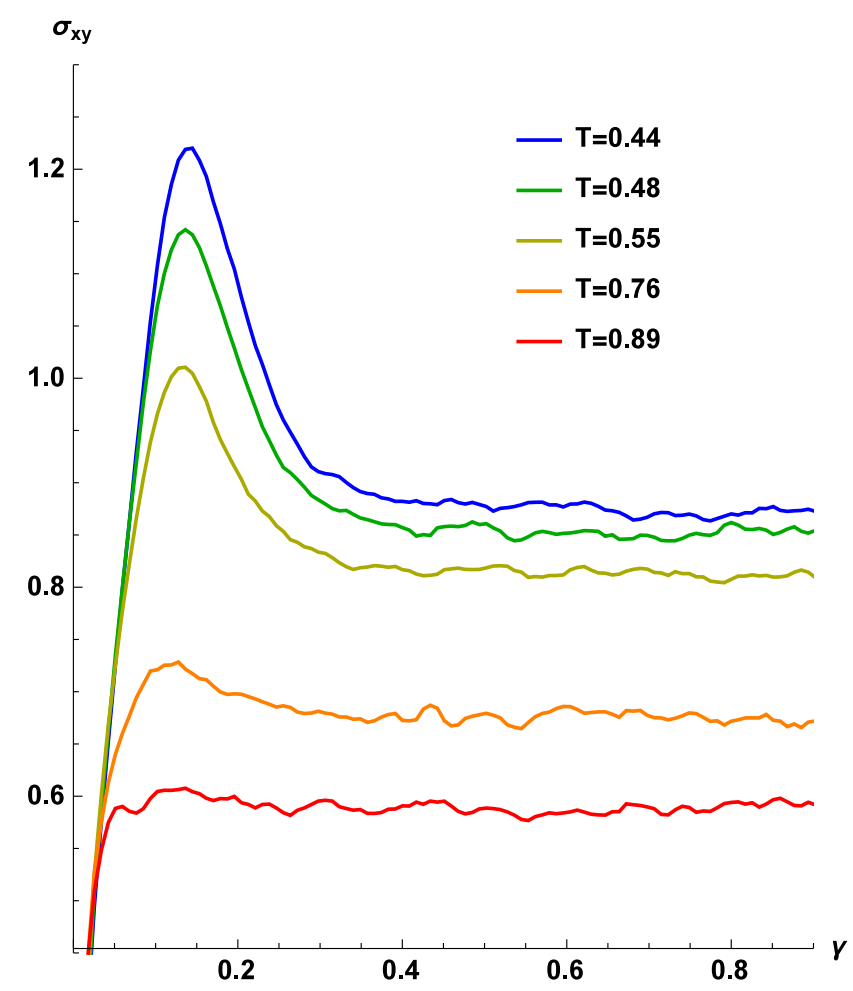

Figure 3. Stress-strain curve for different temperatures $(T=0.44,0.48,0.55,0.69,0.89$ from top to bottom) and applied shear rate $\dot{\gamma}=0.052$ averaged over 40 independent starting configurations.

The steady-state shear stress $\sigma_{\mathrm{ss}}$ that the system attains at very long times after inception of steady shear has been investigated in detail in earlier studies where it was found that $\sigma_{\mathrm{ss}} \sim \dot{\gamma}^{1-\alpha}$ with $\alpha \approx 2 / 3$ for $T>T_{c}$ and not too low shear rates ${ }^{45}$. Moreover, the emergence of a dynamic yield stress occurs in this system at lower temperatures $(T<0.4)^{46}$, beyond the parameter range of the present study. Therefore, all results reported here correspond to a temperature/shear rate regime where the system behaves as a shear-thinning fluid, at least concerning its steady-state properties. 


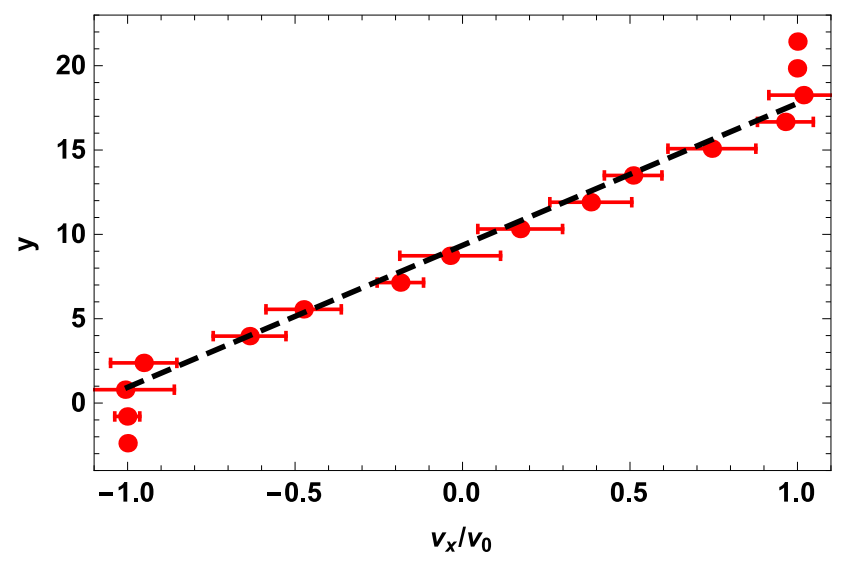

Figure 4. Normalized velocity profile at steady state for $T=0.44$ and $\dot{\gamma}=0.052$. Black line indicates the linear profile $u(y)=\dot{\gamma}(y-L / 2)$ and errorbars correspond to three standard deviations of the velocity in $x$-direction recorded during a period of $t_{v}=0.5$.

\section{Measuring flow profiles}

Contrary to several previous studies on sheared supercooled liquids ${ }^{45,46}$, our procedure does not impose a particular velocity profile onto the system. In order to compute the flow profile, we determine the average velocity in shear direction $\left(v_{x}\right)$ of layers of particles by dividing the simulation box in different bins with a thickness $\Delta y=2$. The bins extend over the entire box length in $x$ - and $z$-direction. By averaging the $x$-component of the velocity of all particles in $i$-th bin, we obtain the average flow velocity of the system in each layer $v_{x}^{i}$. An example of the resulting flow profile is shown in figure 4 .

\section{TRANSIENT SHEAR BANDING}

During the simulations of start-up of steady shear, we not only evaluate the instantaneous shear stress (3) but also monitor the velocity profiles averaged over a short time interval. Results for a representative simulation are shown in Fig. 5. We find that the system quickly assumes a linear flow profile in the initial regime where the shear stress increases roughly proportional to strain (Fig. 5B). However, the linear profile breaks down in the region of the mechanical instability after the stress overshoot where $\mathrm{d} \sigma_{x y} / \mathrm{d} \gamma<0$. In this regime, an inhomogeneous velocity profile known as shear band forms (Fig. 5C) before finally recovering the linear profile at later stages towards the steady state (Fig. 5D). 

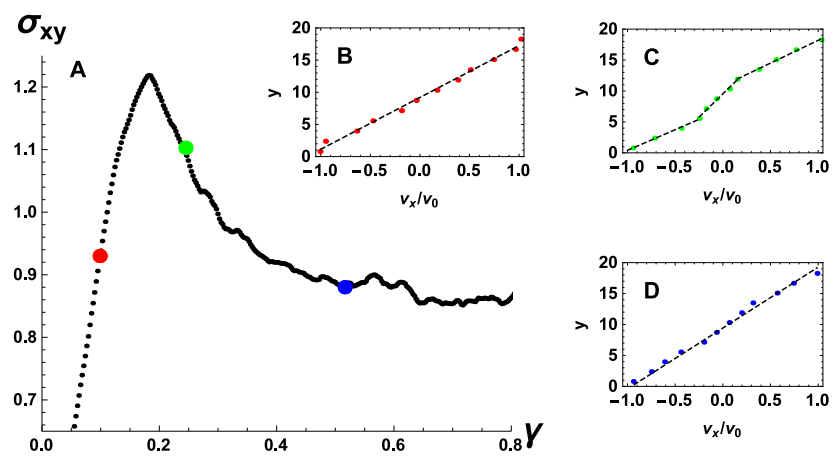

Figure 5. A. Typical stress-strain curve of shear start-up simulation in the deeply supercooled regime and medium shear rate $(T=0.44, \dot{\gamma}=0.052)$ exhibiting a characteristic stress overshoot before leveling off to a steady state value. B-D. Normalized velocity profiles (averaged over short time period $t_{v} \approx 0.1$ ) corresponding to small, intermediate, and large strains as indicated by solid circles in panel A.

We systematically investigate the transient flow profiles for different temperatures and shear rates. Some representative results are shown in Fig. 6. In the vast majority of cases, we find that transient shear bands occur in the center region between the walls.

\section{A. Measuring transient shear banding}

As a measure of shear banding for the system, we compute the goodness of a linear fit ${ }^{47}$ for the linear velocity profile, i.e.

$$
r^{2}=1-s_{r} / s_{t}
$$

where

$$
s_{r}=\sum_{i}\left(v_{x}^{i}-u^{i}\right)^{2} \quad, \quad s_{t}=\sum_{i}\left(v_{x}^{i}-\bar{v}_{x}\right)^{2} .
$$

Here, $u^{i}=u\left(y^{i}\right)$ is the model value of the linear fit for the $i$-th layer and $\bar{v}_{x}$ the average velocity in $x$-direction of the entire system. For the present setup $\bar{v}_{x} \approx 0$. Note, that $r^{2}$ is closely related to the mean-squared deviation from a linear profile via Eq. (5).

In fact, $r^{2}=1$ corresponds to a perfect linear profile, whereas deviation from a linear profile lead to $r^{2}<1$. Since the observed shear bands are not persistent to steady state, we record the coefficient of determination $r^{2}$ of the velocity profile as a function of strain in order to distinguish between banding and fluctuations around the linear profile. The results 

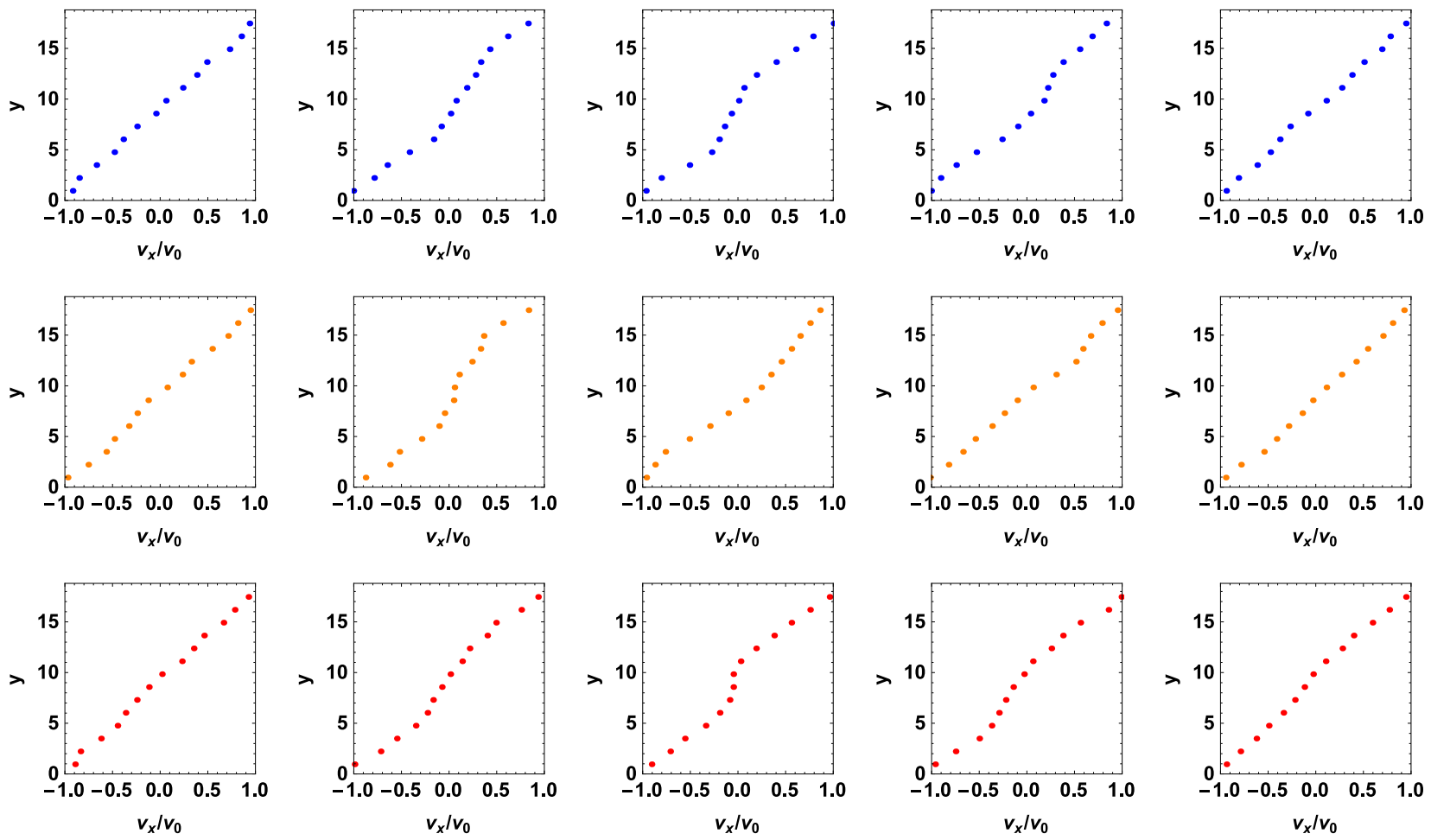

Figure 6. Normalized velocity profiles at $T=0.44$ for different shear rates $\dot{\gamma}=0.032,0.042,0.052$, (top to bottom). The velocities were divided by the absolute value of the wall velocity. Left to right panels demonstrate the evolution of the flow pattern at different stages of the transient regime. Velocity profiles have been averaged over a short time interval $t_{v}=0.1$.

shown in figure 7 confirm that the system initially strongly deviates from a linear profile as the walls start to move but arrives at a linear profile comparable to the steady-state profile at the same time as the maximum of the stress-strain curve is approached. Afterwards, the linear profile breaks down as shear bands form in the system mirrored by a local minimum in the $r^{2}$ versus $t$ curve. The depth of the minimum is significantly larger than fluctuations in the linear profile naturally occurring at steady-state. Visual inspection of the flow-profile shows a piecewise linear profile is formed during this period i.e. shear banding occurs. We identify the slow/fast band right after the stress overshoot, sample the local shear rates in both regions and record a histogram of the data during the full relaxation process starting from the stress-overshoot and ending at steady-state. The time interval of transient shear banding over which we perform this analysis corresponds to half of the width of the drop of $r^{2}$ as a function of time. 


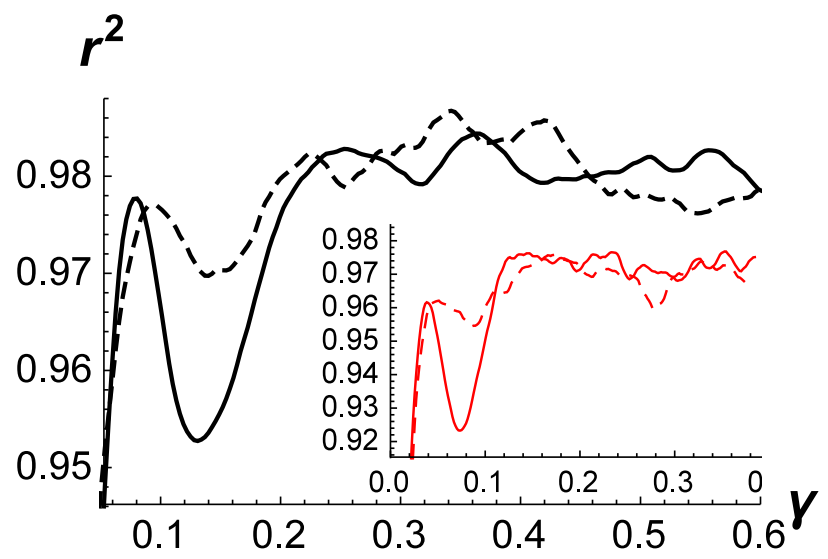

Figure 7. Goodness of a linear fit of the velocity profile during shear start-up simulations for a higher ( $\dot{\gamma}=0.064$, black curves) and a lower $(\dot{\gamma}=0.032$, red curves, inset $)$ shear rate. Dashed lines $T=0.52$, full lines $T=0.46$.

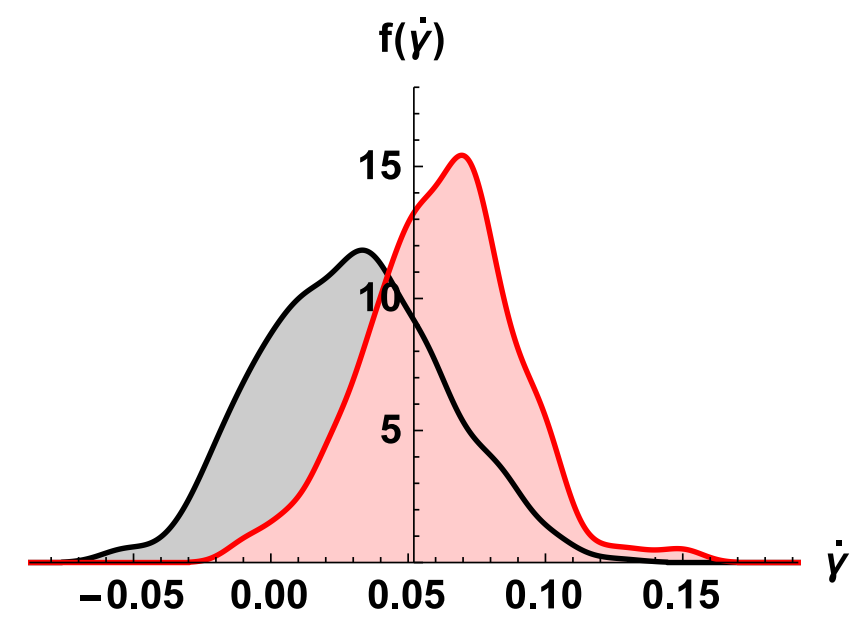

Figure 8. Histograms of local shear rates during the whole lifetime of the shear bands (slow: black, fast: red) for $T=0.44$ and $\dot{\gamma}=0.045$.

The result is depicted in figure 8 showing two separate distributions of the frequency $f(\dot{\gamma})$ around different mean values corresponding to a slow and fast band. We note that both curves coincide before and after the decrease in $r^{2}$ occurs (not shown here). Due to the transient nature of the system, the two histograms overlap over a certain region. We perform a t-test ${ }^{47}$ of the null-hypothesis that the mean values of the fast $\left(\mu_{\text {fast }}\right)$ and slow band $\left(\mu_{\text {slow }}\right)$ are the same $\left(H_{0}: \mu_{\text {fast }}-\mu_{\text {slow }}=0\right)$ against the alternative hypothesis the both regions remain statistically different over the entire relaxation process $\left(H_{1}: \mu_{\text {fast }}-\mu_{\text {slow }} \neq 0\right)$. For 
all investigated samples at which the $r^{2}$-value has a pronounced local minimum in temporal vicinity of the stress overshoot, we find that for both distributions the null-hypothesis has to be rejected within the $95 \%$ confidence interval. Therefore, both distributions are significantly different i.e. the region in which the slower band is formed remains slower during the entire stress-relaxation process and vice versa for the fast region. This supports the conclusion, that stress relaxation throughout the system does not occur randomly but via a breakdown in a locally confined slow and fast band which persist until steady-state.

\section{B. Transient shear banding and stress overshoot}

Although it might not seem surprising at first that glassy systems with spatially inhomogeneous dynamics in the absence of flow also show transient shear banding, it should be noted that these bands form only at sufficiently high shear rates where flow-induced effects are appreciable. We now investigate the relation between the occurrence of transient shear banding and the appearance of a stress overshoot in the stress-strain curve. To that end, first we systematically simulate stress-strain curves for statistically independent samples in the temperature and shear rate $(T-\dot{\gamma})$ plane. We quantify a stress overshoot by the difference of the maximum stress value $\sigma_{\max }$ and the steady state value $\sigma_{\mathrm{ss}}$, normalized by the maximum deviation $\Delta$ of $\sigma_{\mathrm{ss}}$ which we encounter due to stress fluctuations of a single configuration in the steady state regime. This means that values $\left(\sigma_{\max }-\sigma_{\mathrm{ss}}\right) / \Delta \leq 1$ indicate a region in which the overshoot is comparable to the maximum amplitude of the fluctuation in the steady state whereas $\left(\sigma_{\max }-\sigma_{\mathrm{ss}}\right) / \Delta \gg 1$ implies a strongly pronounced overshoot. We chose this normalization such that $\Delta$ does not depend on the sampling time or sample size but enables us to perform single sample evaluations of overshoot and shear-banding. We use this criterion to compute the minimal values $\dot{\gamma}_{\min }(T)$ for which shear banding can be observed. Figure 9 shows the nonequilibrium diagram of normalized stress overshoot and onset of transient shear banding in the $T-\dot{\gamma}$ plane where the latter was set to be at values at which the majority of 40 independent configurations showed a deviation from a linear profile which was stable in the transient regime and significantly larger than thermal fluctuations. A comparison of both quantities shows that the formation of transient shear bands coincides very well with the onset of a stress overshoot. Furthermore, we note that this criterion of $\left(\sigma_{\max }-\sigma_{\mathrm{ss}}\right) / \Delta \geq 1$ is a relatively restrictive criterion for the detection of 
an overshoot meaning that in a larger ensemble averaged stress-strain relation the onset of overshoot formation would be shifted to somewhat higher temperatures whereas the contour lines of Fig. 9 would keep their qualitative form.

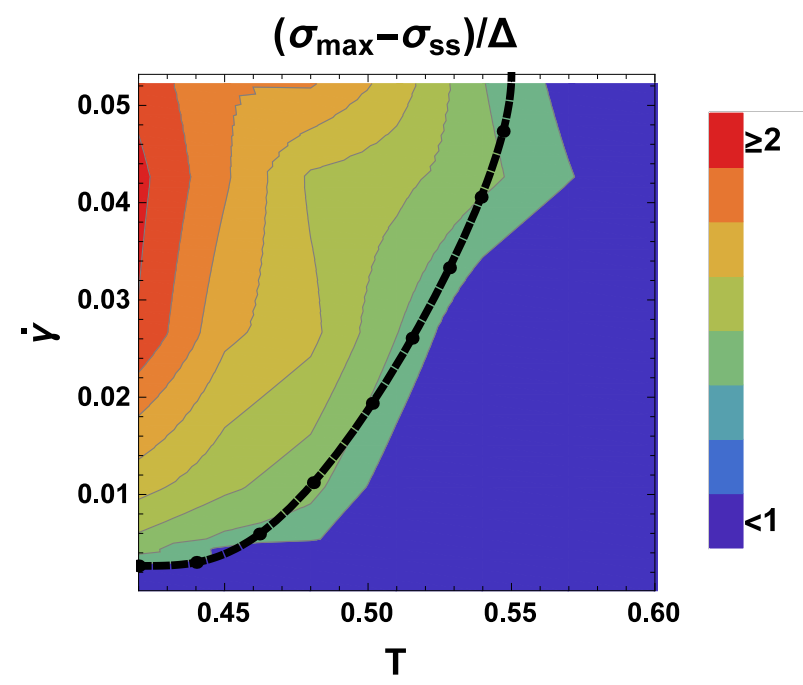

Figure 9. Normalized stress overshoot color-coded as function of temperature and shear rate. While the blue in the bottom right corner (high temperature and low shear rate region) indicates that there is no significant maximum of the stress-strain curve, green to red in the top left corner (low temperature and high shear rate region) indicate the formation of an increasingly pronounced overshoot. Black line marks the minimum $T$ and $\dot{\gamma}$ values at which transient shear bands occur.

Our findings are in qualitative agreement with a recently proposed general criterion for viscoelastic fluids ${ }^{31}$ which states that in the high shear rate limit a mechanical instability emerges whenever a stress overshoot occurs. More, precisely the onset of shear banding is predicted to occur shortly before the overshoot, depending the form of the stress-strain relation during startup. Concerning the underlying mechanism for glassy systems, one plausible explanation refers to spatial heterogeneities that are present in these systems and that manifest themselves in the transient regime.

Some parts of the system adapt more easily to the external perturbation, giving rise to fast shear bands, while other parts with slow dynamics offer more resistance to the applied deformation. They carry an inherent frozen-in rigidity which counteracts the applied deformation and has to be overcome first before reaching steady state. Therefore, regions which form slower bands are subjected to higher tension carrying the majority of stress responsible 
for the overshoot in the transient regime. A distribution of local strain rates around the externally applied rate can be observed with the amplitude being larger for higher applied rates. A large stress accumulated in the system accompanied with a fast relaxation process gives rise to a particularly fast moving shear band which has to be compensated by a slow band to maintain the global shear rate whereas a small accumulated stress value triggers less and "weaker" relaxation events resulting in a smaller difference between the local shear rates in the system. Within the fluidity model $^{25}$, it has similarly been argued that in the shear-thinning regime, regions with higher local shear rate correspond to lower local viscosity and therefore faster relaxation, leading to a positive feedback between local viscosity and local shear rate. Since the initial stress response can be thought to be mainly of elastic nature, one might also refer to phenomenological theories (e.g. Refs. ${ }^{4-52}$ ) that postulate the existence of mesoscopic elements in glassy materials which support elastic stress locally but yield as the stress increases leading to local, cooperative rearrangements of particles that trigger the formation of an inhomogeneous flow profile. As the "weakest" of these elements (e.g. those which can support the least stress) break down, they initiate a local mobilization in the material triggering the formation of an inhomogeneous flow profile. This is in accordance with results for amorphous solids discussed in ${ }^{53}$, where the formation of percolating regions of high mobility at a critical strain was reported.

\section{Local constitutive relation and structure}

Such relaxation processes might be different from those occurring in the nonequilibrium steady state. Therefore, we proceed by investigating whether the global constitutive relation between steady state shear stress and applied shear rate also holds within the shear bands. To answer this question, we compute the local shear rates of all occurring bands by measuring the slope of the velocity profile in each band. Furthermore, we calculate the corresponding local shear stress values by applying the Irving-Kirkwood formula (3) to particles within each shear band. Figure 10 shows that the local shear stresses assumed by the transient shear bands are elevated compared to the corresponding values of the constitutive relation of the system. Despite significant sample-to-sample fluctuations, we consistently observe that slower bands carry higher shear stresses than the fast band for the same local shear rates. 


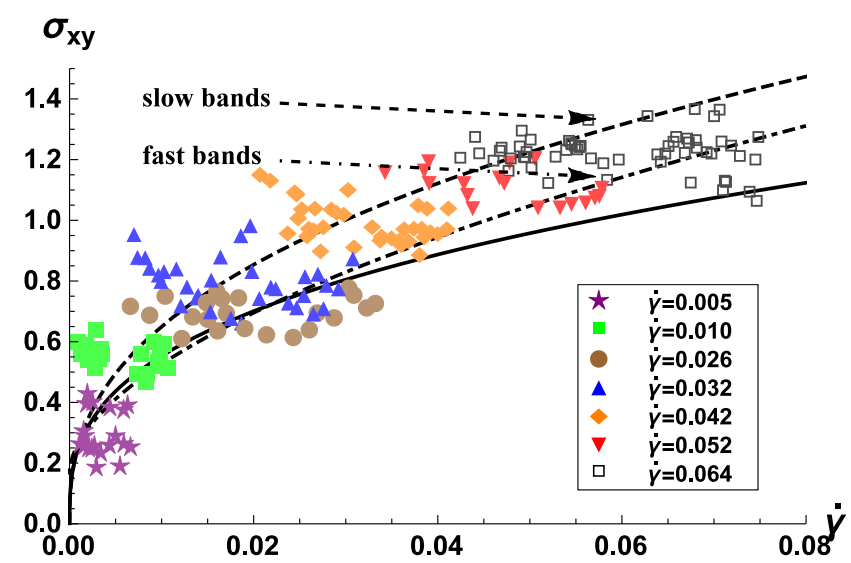

Figure 10. Constitutive curve (steady state shear stress versus applied shear rate) for temperature $T=0.44$ shown as solid black line. Symbols: local shear stress/shear rate values for different configurations measured at time point at which shear banding is most pronounced. Dot-dashed and dashed lines show averages of local shear stress-shear rate values obtained in the fast and slow band, respectively. A careful analysis shows that while the faster bands coincide on average well with the constitutive cure, the slower bands are shifted to significantly higher stress values indicating that the slower bands have not been adapted to the externally applied strain.

We turn to an investigation of the structural origin of the shear bands observed in the transient regime. A simple but efficient tool to investigate the liquid structure is the pair correlation function $g(\mathbf{r})$, its shear-induced angular dependence provides an understanding of the distortion of the local fluid structure ${ }^{54}$. Hence, the pair correlation function is expanded into the spherical harmonics $Y_{l m}^{*}(\hat{\mathbf{r}})$

$$
g(\mathbf{r})=g_{s}(r)+\sum_{l=1}^{\infty} \sum_{m=-l}^{l} g_{l m}(r) Y_{l m}^{*}(\hat{\mathbf{r}}),
$$

where $\hat{\mathbf{r}}$ is the normalized position vector and $g_{s}(r)=1 /(4 \pi) \int g(\mathbf{r}) \mathrm{d} \hat{\mathbf{r}}$ being the isotropic contribution to the pair correlation function. It has been shown that the dominant contribution (to first order in the shear rate) is given by ${ }^{55}$

$$
g_{22}(r)=\frac{15}{4 \pi} \int \hat{r}_{x} \hat{r}_{y} g(\mathbf{r}) \mathrm{d} \hat{\mathbf{r}} .
$$

The peaks of $g_{22}$ increase with increasing shear rate due to enhanced structural distortions ${ }^{55}$. In previous studies $g_{22}$ has been used to monitor the structural changes during shear in supercooled liquid both in simulations and experimental work. ${ }^{42,56}$. We follow a similar route 
and investigate $g_{22}$ as a simple structural indicator in the slow and fast band separately. The steady state form of $g_{22}$ coincides rather well with the signal from the fast shear bands

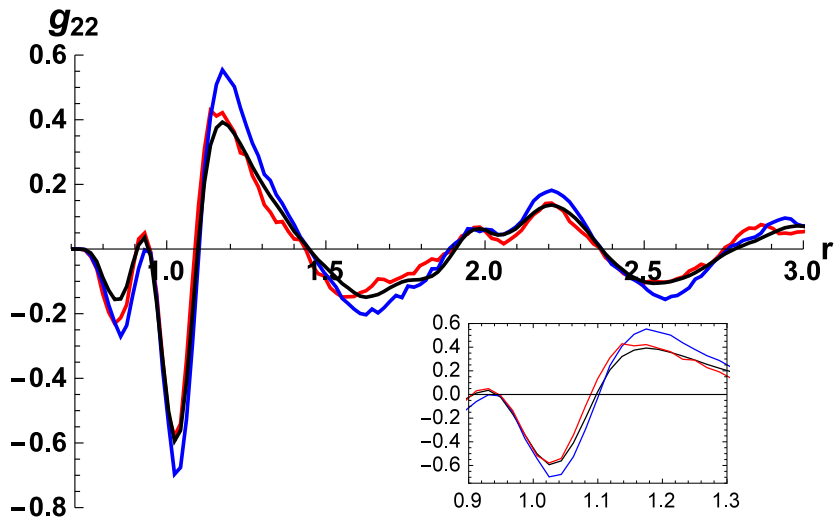

Figure 11. The pair correlation function $g_{22}$, Eq. (7), at temperature $T=0.44$ and $\dot{\gamma}=0.052$ in steady state (black), fast shear band (red) and slow shear band (blue). Inset shows the same data but zoomed into the first two peaks. While the fast band structure coincides rather well with steady state data, the signal from the slow shear band shows significantly larger amplitudes i.e. the elevated stresses in the slower bands as seen in fig. 10 have structural origin mirrored by a more pronounced distortion of the nearest neighbour shells of particles.

in the transient regime, whereas we observe more pronounced peaks in the slower band (see fig. 11). Our findings imply that the fast band is well adapted to the applied shear even in the transient regime. However, the local structure in the slow bands is distorted more strongly than in the steady state. The function $g_{22}$ also determines the (configurational) shear stress in the system via

$$
\left\langle\sigma_{x y}\right\rangle=\frac{2 \pi \rho^{2}}{3} \sum_{i, j} \frac{N_{i} N_{j}}{N^{2}} \int \frac{\mathrm{d} \phi_{i j}(r)}{\mathrm{d} r} r^{3} g_{22}^{i j}(r) \mathrm{d} r,
$$

where $N_{i}$ denotes the number of particles of type $i$ and the superscript of $g_{22}$ indicates that the $i j$-interactions are taken into account separately. Therefore, fig. 11 shows the structural origin of the larger shear stresses carried by the slow band.

Finally, we investigate the local viscous dissipation rate $d=\sigma_{x y}^{1} \dot{\gamma}^{1}$ in both shear bands separately, where the superscript emphasizes the local nature of the quantities under consideration. Surprisingly, we find that the local dissipation in each band is approximately the same in the slow and fast band (see figure 12). Interestingly, minimal dissipation arguments 
seem rather successful in predicting shear bands in granular material ${ }^{57}$ and the present observation on equal dissipation rises the question whether a similar minimal dissipation-like argument could be derived for transient banding.

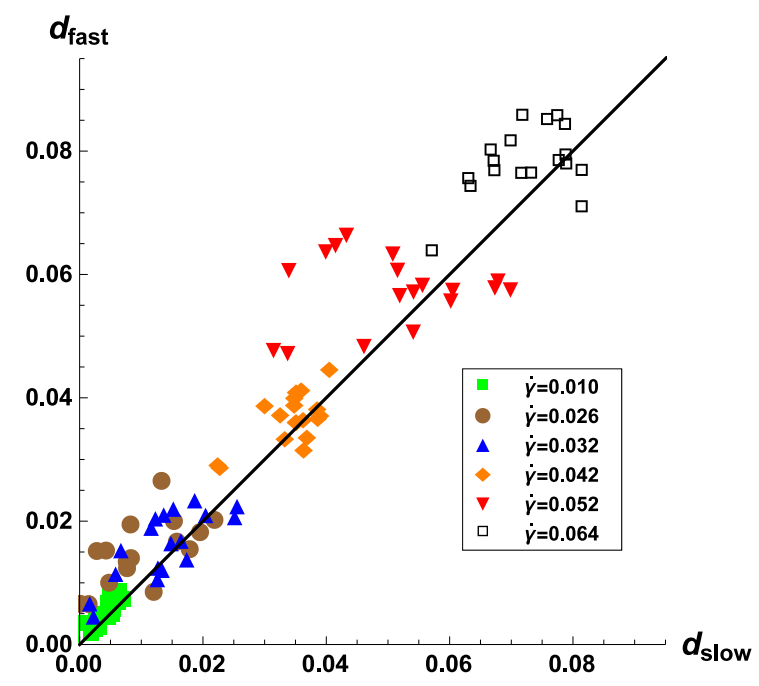

Figure 12. Local dissipation rate $\sigma_{x y}^{1} \dot{\gamma}^{1}$ in the slow and fast band for $T=0.44$. Different symbols correspond to different applied shear rates (same as in figure 4). The line corresponds to $d_{\text {fast }}=$ $d_{\text {slow }}$.

\section{CONCLUSIONS}

To summarize, we have reported the first observation of transient shear banding in a molecular model of supercooled liquids. We found that the occurrence of these shear bands coincides well with the appearance of a stress overshoot over the entire temperature range in the supercooled regime. These observations are in qualitative agreement with predictions for general viscoelastic liquids ${ }^{31}$. We suggest that parts of the system with predominant fast dynamics adapt more quickly to the imposed deformation and form the fast shear band. The other part of the system with slower dynamics is less able to adapt its structure and forms the slow band that presents more resistance to the flow and thus accumulates higher stresses. In principle, it is possible that also more complex flow patterns form during this process. While we found some initial indications pointing in this direction, further studies of larger systems are needed to explore this issue thoroughly. There is currently little understanding about the selection of shear rates but our results indicate that local 
dissipation is very similar in both bands, offering a possible route for future theoretical development. It is worth to note that mode coupling theory ${ }^{10,11}$ provides detailed quantitative predictions also for the transient regime but assumes a homogeneous velocity profile. In the light of the present results, it would be interesting to understand whether extensions of this theory allowing for shear banding will lead to more accurate predictions. As this is the first molecular-based discussion linking transient shear band formation, stress overshoot and local structure in the start-up of steady shear in supercooled liquids, several questions remain open. For instance, it is unclear whether the relaxation events in the slower bands differ qualitatively from those occurring in the fast band. While the latter seem to be rather similar to those in the steady state of the driven system, relaxation in the slow band could show more similarities to elastic deformations in amorphous solids. In this context we note that recent studies indicate the presence of long-ranged Eshelby-like patterns in the local strain correlations of the system (see e.g. Refs. ${ }^{58,59}$ ). These Eshelby fields are assumed to originate from localized strain changes which give rise to localized forces influencing the underlying inherent structure, persist even well above the glass transition temperature in the supercooled regime and seem to play a crucial role in the Newtonian to shear thinning cross-over. ${ }^{58}$ The presence of these Eshelby fields and a possible difference in the slow and fast band in transient flow would be of high interest but is beyond the scope of this paper. These questions are left for future research.

\section{ACKNOWLEDGEMENTS}

We thank Hans Christian Öttinger for insightful discussions and gratefully acknowledge the Swiss National Science Foundation for providing funding under Grant No. 200021_134626. PI is supported by EU FP7-MC-CIG grant no. 631233.

\section{REFERENCES}

${ }^{1}$ W. K. Kegel and A. van Blaaderen, "Direct observation of dynamical heterogeneities in colloidal hard-sphere suspensions," Science 287, 290-293 (2000). 
${ }^{2}$ E. R. Weeks, J. C. Crocker, A. C. Levitt, A. Schofield, and D. A. Weitz, "Threedimensional direct imaging of structural relaxation near the colloidal glass transition," Science 287, 627-631 (2000).

${ }^{3}$ T. Narumi, S. V. Franklin, K. W. Desmond, M. Tokuyama, and E. R. Weeks, "Spatial and temporal dynamical heterogeneities approaching the binary colloidal glass transition," Soft Matter 7, 1472-1482 (2011).

${ }^{4}$ R. Candelier, A. Widmer-Cooper, J. K. Kummerfeld, O. Dauchot, G. Biroli, P. Harrowell, and D. R. Reichman, "Spatiotemporal Hierarchy of Relaxation Events, Dynamical Heterogeneities, and Structural Reorganization in a Supercooled Liquid," Phys. Rev. Lett. 105, 135702 (2010).

${ }^{5}$ R. Besseling, E. R. Weeks, A. B. Schofield, and W. C. K. Poon, "Three-dimensional imaging of colloidal glasses under steady shear," Phys. Rev. Lett. 99, 028301 (2007).

${ }^{6}$ P. Schall, D. A. Weitz, and F. Spaepen, "Structural rearrangements that govern flow in colloidal glasses," Science 318, 1895-1899 (2007).

${ }^{7}$ R. Zondervan, T. Xia, H. van der Meer, C. Storm, F. Kulzer, W. van Saarloos, and M. Orrit, "Soft glassy rheology of supercooled molecular liquids," Proceedings of the National Academy of Sciences of the United States of America 105, 4993-4998 (2008).

${ }^{8}$ J. M. Brader, M. Siebenbuerger, M. Ballauff, K. Reinheimer, M. Wilhelm, S. J. Frey, F. Weysser, and M. Fuchs, "Nonlinear response of dense colloidal suspensions under oscillatory shear: Mode-coupling theory and Fourier transform rheology experiments," Phys. Rev. E 82, 061401 (2010).

${ }^{9}$ J. M. Brader, M. E. Cates, and M. Fuchs, "First-principles constitutive equation for suspension rheology," Phys. Rev. E 86, 138301 (2012).

${ }^{10} \mathrm{M}$. Laurati et al., "Transient dynamics in dense colloidal suspensions under shear: shear rate dependence," J. Phys.: Condens. Mat. 24, 464104 (2012).

${ }^{11}$ C. P. Amann, M. Siebenbürger, M. Krüger, F. Weysser, M. Ballauff, and M. Fuchs, "Overshoots in stress-strain curves: Colloid experiments and schematic mode coupling theory," J. Rheol. 57, 149-175 (2013).

${ }^{12}$ J. Zausch, J. Horbach, M. Laurati, S. U. Egelhaaf, J. M. Brader, T. Voigtmann, and M. Fuchs, "From equilibrium to steady state: the transient dynamics of colloidal liquids under shear," Journal of Physics: Condensed Matter 20, 404210 (2008). 
${ }^{13}$ F. Varnik, L. Bocquet, J.-L. Barrat, and L. Berthier, "Shear Localization in a Model Glass," Phys. Rev. Lett. 90, 095702 (2003).

${ }^{14}$ V. Chikkadi, D. M. Miedema, M. T. Dang, B. Nienhuis, and P. Schall, "Shear banding of colloidal glasses: Observation of a dynamic first-order transition," Phys. Rev. Lett. 113, 208301 (2014).

${ }^{15}$ P. D. Olmsted, "Perspectives on shear banding in complex fluids," Rheol. Acta 47, 283-300 (2008).

${ }^{16}$ J. D. Martin and Y. Thomas Hu, "Transient and steady-state shear banding in aging soft glassy materials," Soft Matter 8, 6940-6949 (2012).

${ }^{17}$ T. Divoux, C. Barentin, and S. Manneville, "From stress-induced fluidization processes to herschel-bulkley behaviour in simple yield stress fluids," Soft Matter 7, 8409-8418 (2011).

${ }^{18}$ T. Divoux, D. Tamarii, C. Barentin, and S. Manneville, "Transient shear banding in a simple yield stress fluid," Phys. Rev. Lett. 104, 208301 (2010).

${ }^{19}$ X. Illa, A. Puisto, A. Lehtinen, M. Mohtaschemi, and M. J. Alava, "Transient shear banding in time-dependent fluids," Phys. Rev. E 87, 022307 (2013).

${ }^{20}$ J. M. Adams, S. M. Fielding, and P. D. Olmsted, "Transient shear banding in entangled polymers: A study using the rolie-poly model," Journal of Rheology 55, 1007-1032 (2011), http://dx.doi.org/10.1122/1.3610169.

${ }^{21}$ M. L. Manning, E. G. Daub, J. S. Langer, and J. M. Carlson, "Rate-dependent shear bands in a shear-transformation-zone model of amorphous solids," Phys. Rev. E 79, 016110 (2009).

${ }^{22}$ Y. Shi, M. B. Katz, H. Li, and M. L. Falk, "Evaluation of the disorder temperature and free-volume formalisms via simulations of shear banding in amorphous solids," Phys. Rev. Lett. 98, 185505 (2007).

${ }^{23}$ A. R. Hinkle and M. L. Falk, "A small-gap effective-temperature model of transient shear band formation during flow," Journal of Rheology 60, 873-882 (2016).

${ }^{24}$ J. S. Langer and T. Egami, "Glass dynamics at high strain rates," Phys. Rev. E 86, 011502 (2012).

${ }^{25}$ A. Lehtinen, A. Puisto, X. Illa, M. Mohtaschemi, and M. J. Alava, "Transient shear banding in viscoelastic maxwell fluids," Soft Matter 9, 8041-8049 (2013).

${ }^{26}$ E. A. Jagla, "Strain localization driven by structural relaxation in sheared amorphous solids," Phys. Rev. E 76, 046119 (2007). 
${ }^{27}$ E. A. Jagla, "Shear band dynamics from a mesoscopic modeling of plasticity," Journal of Statistical Mechanics: Theory and Experiment 2010, P12025 (2010).

${ }^{28}$ S. M. Fielding, "Shear banding in soft glassy materials," Rep. Prog. Phys. 77, 102601 (2014).

${ }^{29} \mathrm{~S}$. M. Fielding, "Triggers and signatures of shear banding in steady and time-dependent flows," Journal of Rheology 60, 821-834 (2016).

${ }^{30}$ R. L. Moorcroft, M. E. Cates, and S. M. Fielding, "Age-dependent transient shear banding in soft glasses," Phys. Rev. Lett. 106, 055502 (2011).

${ }^{31}$ R. L. Moorcroft and S. M. Fielding, "Criteria for Shear Banding in Time-Dependent Flows of Complex Fluids," Phys. Rev. Lett. 110, 086001 (2013).

${ }^{32}$ J. Cao and A. E. Likhtman, "Shear banding in molecular dynamics of polymer melts," Phys. Rev. Lett. 108, 028302 (2012).

${ }^{33}$ G. P. Shrivastav, P. Chaudhuri, and J. Horbach, "Heterogeneous dynamics during yielding of glasses: Effect of aging," Journal of Rheology 60, 835-847 (2016).

${ }^{34} \mathrm{P}$. Chaudhuri and J. Horbach, "Onset of flow in a confined colloidal glass under an imposed shear stress," Phys. Rev. E 88, 040301 (2013).

${ }^{35} \mathrm{~W}$. Kob and H. C. Andersen, "Testing mode-coupling theory for a supercooled binary lennard-jones mixture i: The van hove correlation function," Phys. Rev. E 51, 4626-4641 (1995).

${ }^{36}$ D. J. Evans and G. P. Morriss, "Shear thickening and turbulence in simple fluids," Phys. Rev. Lett. 56, 2172-2175 (1986).

${ }^{37}$ P. Español, "Hydrodynamics from dissipative particle dynamics," Phys. Rev. E 52, 1734$1742(1995)$.

${ }^{38}$ P. J. Hoogerbrugge and J. M. V. A. Koelman, "Simulating microscopic hydrodynamic phenomena with dissipative particle dynamics," EPL (Europhysics Letters) 19, 155 (1992).

${ }^{39}$ S. Plimpton, "Fast parallel algorithms for short-range molecular dynamics," J. Comp. Phys. 117, 1 - 19 (1995).

${ }^{40}$ F. Varnik, L. Bocquet, and J.-L. Barrat, "A study of the static yield stress in a binary Lennard-Jones glass," J. Chem. Phys. 120, 2788-2801 (2004).

${ }^{41}$ J. H. Irving and J. G. Kirkwood, "The statistical mechanical theory of transport processes. iv. the equations of hydrodynamics," J. Chem. Phys. 18, 817-829 (1950). 
${ }^{42}$ J. Zausch and J. Horbach, "The build-up and relaxation of stresses in a glass-forming softsphere mixture under shear: A computer simulation study," EPL (Europhysics Letters) 88, 60001 (2009).

${ }^{43}$ K. A. Erk, J. D. Martin, Y. T. Hu, and K. R. Shull, "Extreme strain localization and sliding friction in physically associating polymer gels," Langmuir 28, 4472-4478 (2012), pMID: 22300309, http://dx.doi.org/10.1021/la204592r.

${ }^{44}$ A. Agrawal, H.-Y. Yu, A. Sagar, S. Choudhury, and L. A. Archer, "Molecular origins of temperature-induced jamming in self-suspended hairy nanoparticles," Macromolecules 49, 8738-8747 (2016), http://dx.doi.org/10.1021/acs.macromol.6b01280.

${ }^{45}$ L. Berthier and J.-L. Barrat, "Nonequilibrium dynamics and fluctuation-dissipation relation in a sheared fluid," J. Chem. Phys. 116, 6228-6242 (2002).

${ }^{46}$ F. Varnik and O. Henrich, "Yield stress discontinuity in a simple glass," Phys. Rev. B 73, 174209 (2006).

${ }^{47}$ W. H. Press, S. A. Teukolsky, W. T. Vetterling, and B. P. Flannery, Numerical Recipes in C (2Nd Ed.): The Art of Scientific Computing (Cambridge University Press, New York, NY, USA, 1992).

${ }^{48}$ G. Picard, A. Ajdari, L. Bocquet, and F. Lequeux, "Simple model for heterogeneous flows of yield stress fluids," Phys. Rev. E 66, 051501 (2002).

${ }^{49}$ L. Bocquet, A. Colin, and A. Ajdari, "Kinetic theory of plastic flow in soft glassy materials," Phys. Rev. Lett. 103, 036001 (2009).

${ }^{50}$ V. Mansard, A. Colin, P. Chauduri, and L. Bocquet, "A kinetic elasto-plastic model exhibiting viscosity bifurcation in soft glassy materials," Soft Matter 7, 5524-5527 (2011).

${ }^{51}$ O. Pouliquen and Y. Forterre, "A non-local rheology for dense granular flows," Phil. Trans. R. Soc. A 367, 5091-5107 (2009).

${ }^{52}$ P. Sollich, F. Lequeux, P. Hébraud, and M. E. Cates, "Rheology of soft glassy materials," Phys. Rev. Lett. 78, 2020-2023 (1997).

${ }^{53}$ G. P. Shrivastav, P. Chaudhuri, and J. Horbach, "Yielding of glass under shear: A directed percolation transition precedes shear-band formation," Phys. Rev. E 94, 042605 (2016).

${ }^{54} \mathrm{~S}$. Hess and H. Hanley, "Pressure tensor and viscosity coefficients of a soft sphere liquid under shear," Inter. J. of Thermophys. 4, 97-114 (1983).

${ }^{55}$ H. J. M. Hanley, J. C. Rainwater, and S. Hess, "Shear-induced angular dependence of the liquid pair correlation function," Phys. Rev. A 36, 1795-1802 (1987). 
${ }^{56}$ C. P. Amann, D. Denisov, M. T. Dang, B. Struth, P. Schall, and M. Fuchs, "Shear-induced breaking of cages in colloidal glasses: Scattering experiments and mode coupling theory," The Journal of Chemical Physics 143, 034505 (2015), http://dx.doi.org/10.1063/1.4926932.

${ }^{57}$ T. Unger, J. Török, J. Kertész, and D. E. Wolf, "Shear Band Formation in Granular Media as a Variational Problem," Phys. Rev. Lett. 92, 214301 (2004).

${ }^{58} \mathrm{~J}$. Chattoraj and A. Lemaître, "Elastic signature of flow events in supercooled liquids under shear," Phys. Rev. Lett. 111, 066001 (2013).

${ }^{59}$ B. Illing, S. Fritschi, D. Hajnal, C. Klix, P. Keim, and M. Fuchs, "Strain pattern in supercooled liquids," Phys. Rev. Lett. 117, 208002 (2016). 